\title{
Diagnostic evaluation of papillary lesions of the breast on core biopsy
}

\author{
Nirmala Pathmanathan ${ }^{1,2,3,4,6}$, Ann-Flore Albertini ${ }^{1,6}$, Pamela J Provan ${ }^{3,4,5}$, \\ Jane S Milliken ${ }^{1}$, Elizabeth L Salisbury ${ }^{1,2}$, A Michael Bilous ${ }^{1,4}$, Karen Byth ${ }^{3}$ and \\ Rosemary L Balleine ${ }^{3,4,5}$ \\ ${ }^{1}$ Department of Tissue Pathology, Institute of Clinical Pathology and Medical Research, Sydney West Area \\ Health Service, Westmead, NSW, Australia; ${ }^{2}$ BreastScreen Greater Western Sydney, Westmead, NSW, \\ Australia; ${ }^{3}$ Westmead Millennium Institute, Westmead, NSW, Australia; ${ }^{4}$ Sydney Medical School-Western, \\ University of Sydney, Westmead, NSW, Australia and ${ }^{5}$ Translational Oncology, Sydney West Area Health \\ Service and Westmead Institute for Cancer Research, Westmead, NSW, Australia
}

\begin{abstract}
The management of asymptomatic intraductal papillary lesions of the breast diagnosed on core biopsy poses a challenge for patients and clinicians, as the distinction between common benign lesions and atypical or malignant varieties may be difficult without formal excision. The aim of this study was to determine whether a combination of histopathologic and biomarker features could be used to accurately identify benign papillary lesions on core biopsy. An inclusive group of 127 excised papillary lesions was characterized by detailed histopathologic review and immunohistochemical staining for the basal markers cytokeratin 5/6 (CK5/6) and P63 and the proliferation marker Ki67. Comparison of benign, atypical, and malignant lesions revealed that the combination of broad, sclerotic fibrovascular cores, and epithelial CK5/6 staining was most commonly seen in benign papillomas. Ki67 staining revealed striking intralesional heterogeneity, but there was no difference between the high scores of benign, atypical, or malignant lesions $(P=0.173)$. In a non-overlapping set of 42 cases, a binary classifier specifying benign lesions on the basis of thick fibrovascular cores and epithelial CK5/6 staining on core biopsy gave an overall misclassification rate of $4 / 42(10 \%)$ when compared with the final excision diagnosis. Misclassified cases included 2/27 lesions ultimately diagnosed as benign and 2/2 atypical papillomas. All malignant lesions $(n=13)$ were correctly assigned. The combined assessment of fibrovascular core thickness and CK5/6 staining on core biopsy distinguished benign from malignant papillary lesions, but did not separate benign from atypical cases. This approach may form a useful addition to the clinicopathologic evaluation of papillary lesions of the breast.
\end{abstract}

Modern Pathology (2010) 23, 1021-1028; doi:10.1038/modpathol.2010.81; published online 14 May 2010

Keywords: papillary; papilloma; breast; pathology core biopsy

Intraductal papillary lesions of the breast are recognized histologically by the presence of fibrovascular cores supporting a variable degree of epithelial proliferation. They are most commonly benign; however, papillary lesions may show premalignant (atypical) histological features and malignant papillary lesions are also recognized including papillary ductal carcinoma in situ (DCIS),

Correspondence: Dr N Pathmanathan, BSc(med), MBBS, FRCPA, Department of Tissue Pathology, Institute of Clinical Pathology and Medical Research, Westmead Hospital, PO Box 533, Westmead, NSW 2145, Australia.

E-mail: nirmala.pathmanathan@swahs.health.nsw.gov.au

${ }^{6}$ These authors contributed equally to this work.

Received 23 December 2009; revised 19 March 2010; accepted 24 March 2010; published online 14 May 2010 intracystic papillary carcinoma, and solid papillary carcinoma. $^{1,2}$

The rate of underdiagnosis of atypia or malignancy following a core biopsy diagnosis of benign papilloma is $>20 \%$ in some reports. $^{3-7}$ Consequently, excision is generally recommended to establish a definitive diagnosis for asymptomatic papillary lesions that are diagnosed on core biopsy following mammographic detection. However, an accurate core biopsy prediction of the final diagnostic category would provide a useful guide to management and may allow further surgery to be avoided for some patients.

The aim of this study was to determine whether benign papillary lesions of the breast could be accurately identified in core biopsies by an assessment of specific histopathologic and/or immunohistochemical 
features. To do this, 127 excised papillary lesions, including benign, atypical, and malignant cases, were examined by detailed histopathology review, staining for basal epithelial markers cytokeratin 5/6 (CK5/6) and p63, and the proliferation marker Ki67. A combination of two features associated with benign lesions, broad sclerotic fibrovascular cores, and positive epithelial staining for CK5/6, was then examined in diagnostic core biopsies from a non-overlapping group of 42 cases, to determine whether a final excision diagnosis of benign papilloma could be predicted using this approach.

\section{Materials and methods}

\section{Patient Samples}

Cases were papillary lesions of the breast diagnosed on breast excision specimens and held in pathology archives at the Institute of Clinical Pathology and Medical Research, Westmead Hospital, Australia. An inclusive group of 127 cases formed the initial reviewed cohort and a non-overlapping group of 42 cases that had been diagnosed on core biopsy and subsequently excised, was used to examine performance of the putative predictor of a benign lesion.

This study was conducted with approval from the Human Research Ethics Committee of the Sydney West Area Health Service (Westmead).

\section{Histopathology Review and Diagnostic Classification; Initial Cohort}

For the initial group of 127 excised cases, one to three hematoxylin and eosin (H\&E)-stained sections were selected and reviewed independently by two observers (NP and AA). Individual histopathologic features were scored using a standardized tick-box review form. This included architectural and nuclear features, stromal changes, and the presence of metaplasia as summarized in Table 1. If multiple scores were entered for a feature, the highest score only was used in analysis and is listed in Table 1. Any discrepancies in scoring between observers were considered at conference review and a third observer was consulted if necessary (MB). Consensus observations were used in analysis.

To ensure consistency in diagnostic categories, cases were assigned as benign, atypical, or malignant based on review assessment of architectural features and the myoepithelial cell layer visualized by immunohistochemical staining. Benign lesions were defined as cases showing epithelial cell hyperplasia equivalent to usual ductal hyperplasia or less, in the presence of a continuous myoepithelial cell layer. Malignant lesions were defined as those showing either complete absence of the myoepithelial cell layer, or a uniformly atypical or solid growth pattern in combination with partial absence of the myoepithelial cell layer. Remaining cases were designated atypical. These definitions were derived with reference to published diagnostic criteria for papillary lesions. ${ }^{1,2,8}$

\section{Immunohistochemical Staining and Evaluation}

Immunohistochemical staining for the proliferation marker Ki67 was performed and a dual staining protocol was used to simultaneously evaluate the basal markers CK5/6 and P63.

Immunohistochemical staining was completed using the Ventana BenchMark IHC/ISH Staining Module (Ventana Medical Systems, Tucson, AZ, USA) with the BMK I-VIEW DAB detection kit (Ventana Medical Systems). For Ki67, the primary antibody clone used was SP6 (NeoMarkers, Fremont, CA, USA), at 1:200 dilution in VP Monet Blue (Biocare Medical). For dual P63 + CK5/6 staining, the primary antibody clone used was P63 (NeoMarkers), at 1:200 dilution in VP Monet Blue and detection with UL-VIEW detection kit, followed by Keratin 5/6 (Cellmarque, Rocklin, CA, USA) at 1:100 dilution using UV Alkaline phosphatase detection kit, and chromogen substrate Fast Red/Naphthol. A positive control section was included in each staining run. Cases that did not show positive staining in any tissue compartment were regarded as unsatisfactory and were excluded from analysis.

Nuclear staining for P63 and cytoplasmic staining for CK5/6 were assessed in the myoepithelial cells of the excised papillary lesions. The myoepithelial cell layer was then scored as present, focally or partly absent or absent. In cases with focal or partial absence, the location of the area of loss of staining was recorded as occurring at the stromal epithelial interface, within the lesion or at the lesion periphery. In addition, intralesional epithelial cell staining for CK5/6 was separately recorded as either present or absent.

For Ki67, sections were scanned using a ScanScope CS (Aperio Technologies, Vista, CA, USA), and digital images visualized using ImageScope Version 6 (Aperio Technologies). In view of striking intralesional heterogeneity in Ki67 staining, areas of high and low staining corresponding to a highpowered microscope field $(0.55 \mathrm{~mm}$ in diameter) were separately identified and scored for each case. Positive and negatively stained nuclei were counted using the manual tag function of Image Pro Plus version 5 (Media Cybernetics, Bethesda, MD, USA) and the percentage of nuclei positively stained represented the Ki67 score. Between 357 and 2099 cells were counted in each area.

\section{Evaluation of a Papillary Lesion Classifier in Core Biopsies}

In the second group of 42 cases, one to three H\&Estained sections of diagnostic core biopsies were 
Table 1 Histopathologic features of 127 papillary lesions of the breast

\begin{tabular}{|c|c|c|c|c|c|c|}
\hline Score & Histopathologic features & $\begin{array}{c}A l l \\
\mathrm{n}(\%)\end{array}$ & $\begin{array}{c}\text { Benign } \\
\text { n (\%) }\end{array}$ & $\begin{array}{l}\text { Atypical } \\
\text { n (\%) }\end{array}$ & $\begin{array}{c}\text { Malignant } \\
\text { n (\%) }\end{array}$ & $\mathrm{P}$-value ${ }^{\mathrm{a}}$ \\
\hline & Total number of cases & 127 & 78 & 26 & 23 & \\
\hline & Architectural features $(\mathrm{n}=127)$ & & & & & \\
\hline 1 & Papillary_single layer & $27(21)$ & $26(33)$ & $1(4)$ & $0(0)$ & $<0.001$ \\
\hline 2 & Papillary-stratified & $22(17)$ & $19(24)$ & $2(8)$ & $1(4)$ & \\
\hline 3 & Florid hyperplasia as seen in UDH & $44(35)$ & $33(42)$ & $11(42)$ & $0(0)$ & \\
\hline 4 & Atypical architectural patterns as seen in $\mathrm{ADH}$ & $27(21)$ & $0(0)$ & $12(46)$ & $15(65)$ & \\
\hline \multirow[t]{2}{*}{5} & Solid & $7(6)$ & $0(0)$ & $0(0)$ & $7(30)$ & \\
\hline & Extent of atypia $(\mathrm{n}=127)$ & & & & & \\
\hline 1 & None & $94(74)$ & $78(100)$ & $13(50)$ & $3(13)$ & $<0.001$ \\
\hline 2 & Focal $<30 \%$ of lesion & $8(6)$ & $0(0)$ & $8(31)$ & $0(0)$ & \\
\hline 3 & Focal $<60 \%$ of lesion & $2(2)$ & $0(0)$ & $1(4)$ & $1(4)$ & \\
\hline 4 & Subtotal $<90 \%$ of lesion & $4(3)$ & $0(0)$ & $3(12)$ & $1(4)$ & \\
\hline \multirow[t]{2}{*}{5} & Uniformly atypical & $19(15)$ & $0(0)$ & $1(4)$ & $18(78)$ & \\
\hline & Myoepithelial cell layer $(\mathrm{n}=127)$ & & & & & \\
\hline 1 & Uniformly present & $82(65)$ & $78(100)$ & $4(15)$ & $0(0)$ & $<0.001$ \\
\hline 2 & Focally absent & $26(20)$ & $0(0)$ & $16(62)$ & $10(43)$ & \\
\hline 3 & Partly absent & $7(6)$ & $0(0)$ & 5 (19) & $2(9)$ & \\
\hline \multirow[t]{2}{*}{4} & Completely absent & $12(9)$ & $0(0)$ & $1(4)$ & $11(48)$ & \\
\hline & Nuclear features ( $c f$ normal epithelial cells) $(\mathrm{n}=12$ & & & & & \\
\hline 1 & Small, uniform chromatin & $19(15)$ & $13(17)$ & $6(23)$ & $0(0)$ & $<0.001$ \\
\hline 2 & Larger, chromatin margination, small nucleoli & $94(74)$ & $63(81)$ & $16(62)$ & $15(65)$ & \\
\hline \multirow[t]{2}{*}{3} & Larger, irregular chromatin, prominent nucleoli & $14(11)$ & $2(3)$ & $4(15)$ & $8(35)$ & \\
\hline & Cell borders $(\mathrm{n}=126)$ & & & & & \\
\hline 1 & Distinct & $5(4)$ & $0(0)$ & $0(0)$ & $5(22)$ & $<0.001$ \\
\hline \multirow[t]{2}{*}{2} & Indistinct & $121(96)$ & $77(100)$ & $26(100)$ & $18(78)$ & \\
\hline & Metaplasia $(\mathrm{n}=122)$ & & & & & \\
\hline 1 & None & $76(62)$ & $45(59)$ & $12(50)$ & $19(86)$ & 0.109 \\
\hline 2 & Apocrine & $39(32)$ & $27(36)$ & 9 (38) & $3(14)$ & \\
\hline 3 & Sebaceous & $2(2)$ & $1(1)$ & $1(4)$ & $0(0)$ & \\
\hline 4 & Mucinous & $0(0)$ & $0(0)$ & $0(0)$ & $0(0)$ & \\
\hline \multirow[t]{2}{*}{5} & Other & $5(4)$ & $3(4)$ & $2(8)$ & $0(0)$ & \\
\hline & Fibrovascular cores $(\mathrm{n}=127)$ & & & & & \\
\hline 1 & Thin and arborizing & $83(65)$ & $43(55)$ & $20(77)$ & $20(87)$ & 0.006 \\
\hline \multirow[t]{2}{*}{2} & Broad and sclerotic (or both) & $44(35)$ & $35(45)$ & $6(23)$ & $3(13)$ & \\
\hline & Epithelial entrapment in capsule $(\mathrm{n}=122)$ & & & & & \\
\hline 1 & Present & $60(49)$ & $33(43)$ & $17(71)$ & $10(48)$ & 0.053 \\
\hline \multirow[t]{2}{*}{2} & Absent & $62(51)$ & $44(57)$ & $7(29)$ & $11(52)$ & \\
\hline & Perilesional sclerosis $(\mathrm{n}=127)$ & & & & & \\
\hline 1 & Minimal & $28(22)$ & $18(23)$ & $6(23)$ & 4 (17) & 0.659 \\
\hline 2 & Moderate & $76(60)$ & $47(60)$ & $13(50)$ & $16(70)$ & \\
\hline \multirow[t]{2}{*}{3} & Prominent & $23(18)$ & $13(17)$ & $7(27)$ & $3(13)$ & \\
\hline & Surrounding breast changes $(\mathrm{n}=113)$ & & & & & \\
\hline 1 & Normal & $39(35)$ & $28(41)$ & $7(28)$ & $4(20)$ & 0.042 \\
\hline 2 & Usual ductal hyperplasia & $17(15)$ & $11(16)$ & $4(16)$ & $2(10)$ & \\
\hline 3 & Atypical ductal hyperplasia (ADH) & $3(3)$ & $0(0)$ & $1(4)$ & $2(10)$ & \\
\hline 4 & Columnar cell lesion ${ }^{\mathrm{b}}$ & $43(38)$ & $24(35)$ & $9(36)$ & $10(50)$ & \\
\hline 5 & Lobular carcinoma in situ & $0(0)$ & $0(0)$ & $0(0)$ & $0(0)$ & \\
\hline 6 & DCIS & $4(4)$ & $0(0)$ & $3(12)$ & $1(5)$ & \\
\hline \multirow[t]{2}{*}{0} & Other & $7(6)$ & $5(7)$ & $1(4)$ & $1(5)$ & \\
\hline & Epithelial CK5/6 staining $(\mathrm{n}=122)$ & & & & & \\
\hline 1 & Absent & $29(24)$ & $2(3)$ & $7(30)$ & $20(87)$ & $<0.001$ \\
\hline 2 & Present & $93(76)$ & $74(97)$ & $16(70)$ & $3(13)$ & \\
\hline
\end{tabular}

${ }^{\mathrm{a}}$ Fisher's exact test.

${ }^{\mathrm{b}} \mathrm{ADH}$ was not separately recorded for any cases in this category.

reviewed by a single observer (NP). Fibrovascular cores were scored as either (1) including broad, sclerotic cores or (2) thin, arborizing cores only. A single stain for CK5/6 was also performed on core biopsy sections (Cellmarque) with the primary antibody at 1:200 dilution detected with the UL-VIEW 
detection kit using VP Monet Blue (BioCare Medical). Staining for CK5/6 in the epithelial component of the lesions was recorded as (1) present or (2) absent. Cases that showed a combination of broad sclerotic cores and CK5/6 staining on core biopsy were allocated to the benign category. This categorization was then compared with the final excision diagnosis derived from original pathology reports. The final diagnosis was benign in 27 cases, atypical in two cases (atypical papilloma \pm atypical ductal hyperplasia) and malignant in 13 cases (including six cases of DCIS only and seven cases with invasive carcinoma).

\section{Statistical Analysis}

Statistical analyses were performed using SPSS for Windows Version 17.0 (SPSS, Chicago, IL, USA). Two-tailed tests with a significance level of $5 \%$ were used throughout. Fisher's exact test was used to test for associations between histopathologic features and categories. Kruskal-Wallis non-parametric analysis of variance was used to test the distribution of Ki67 positivity between categories.

\section{Results}

\section{Histopathologic Features of Papillary Lesions in Surgical Excision Specimens}

Histopathologic features were systematically documented in 127 excised papillary lesions of the breast (Table 1). A combination of architectural features and completeness of the myoepithelial cell layer was used to specify benign, atypical, and malignant categories, resulting in 78 lesions benign lesions (61\%), 26 atypical $(20 \%)$, and $23(18 \%)$ malignant.

Benign papillomas were characterized by a continuous myoepithelial cell layer surmounted by single epithelial cell layer or epithelial hyperplasia with features of usual epithelial hyperplasia (Figure 1ai-ii). Broad, sclerotic fibroepithelial cores were seen in 35 cases (45\%), whereas 43 (55\%) cases showed thin arborizing fibrovascular cores only. Benign lesions also tended to show a patchy or mosaic pattern of CK5/6 staining of epithelial cells (Figure 1aiii) consistent with a heterogeneous mix of epithelial cell types.

Atypical lesions generally showed a greater degree of epithelial proliferation with florid hyperplasia and atypical architectural patterns that frequently occupied only a portion of the lesion. The myoepithelial cell layer was uniformly present in only $4 / 26$ cases ( $15 \%$ ). In comparison with benign papillomas, a greater proportion of atypical lesions showed only thin, arborizing fibrovascular cores (77\%). Epithelial cell staining for CK5/6 was present in $70 \%$ of cases. It was further noted that areas of focal atypical architecture were often associated with a focal loss of CK5/6 staining (Figure 1b).
Malignant lesions had more complex architecture involving most or all of the lesion, and seven cases $(30 \%)$ had a solid epithelial growth pattern. There was complete absence of the myoepithelial cell layer (both at the intralesional epithelial stromal interface and at the lesion periphery) in 11/23 lesions (48\%), and partial or focal absence of myoepithelial cells in the remainder. The majority of malignant papillary lesions had only thin and arborizing fibrovascular cores $(87 \%)$. Epithelial cell staining for CK5/6 was present in only $3 / 23$ cases (13\%) (Figure 1c).

A range of changes in breast tissue surrounding the papillary lesions were observed in the reviewed sections. In particular, it was noted that concomitant columnar cell lesions were relatively common.

\section{Cellular Proliferation Assessed by Ki67 Staining}

There was marked area-to-area variation in Ki67 staining within papillary lesions. To take account of this in evaluation of cellular proliferation, separate Ki67 counts were made in low- and high-staining areas for each case. There was a mean difference of $12.6 \%( \pm 9.5 \%)$ between high- and low-staining areas across the cohort. This difference was not significantly different between benign, atypical, or malignant papillary lesions $(P=0.240$, KruskalWallis) (Figure 2a). There was no significant difference in Ki67 high scores $(P=0.173$, KruskalWallis) between these categories (Figure 2b).

\section{Predictive Assessment of Benign and Malignant Papillary Lesions}

Apart from the architectural and myoepithelial cell layer characteristics that were used to define the benign, atypical, and malignant subcategories of papillary lesion, nuclear features $(P<0.001$, Fisher's exact test), distinct cell borders $(P<0.001$, Fisher's exact test), presence of broad, sclerotic fibrovascular cores $(P=0.006$, Fisher's exact test $)$ and epithelial staining for CK5/6 $(P<0.001$, Fisher's exact test) were significantly different between the categories. Notwithstanding these differences, none of these individual features were sufficiently distinctive to act as an indicator of a benign lesion. However, it was noted that the combination of broad, sclerotic fibrovascular cores and epithelial CK5/6 staining was significantly different between benign and atypical/malignant lesions $(P<0.001$, Fisher's exact test) with $34 / 37$ cases (92\%) of lesions showing both of these features belonging to the benign group compared with $1 / 24(4 \%)$ cases with only thin cores and no CK5/6 staining present (Figure 2c). On this basis, it was postulated that identification of both broad, sclerotic fibrovascular cores and CK5/6 staining on core biopsy may predict a benign lesion at excision. 

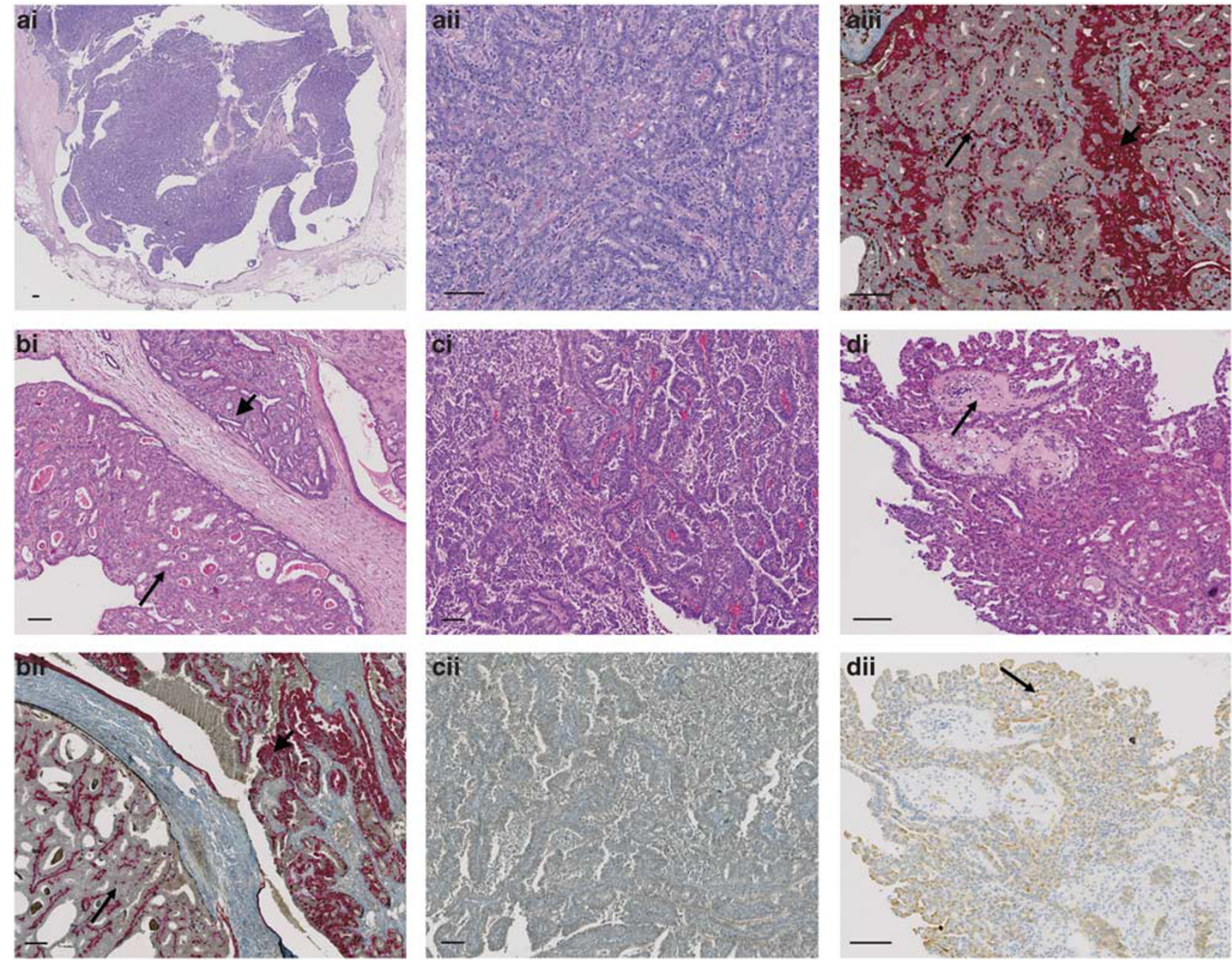

Figure 1 (ai-ii) Benign papilloma (H\&E). (aiii) Dual CK5/6 (red) and P63 (brown) immunohistochemical staining of benign papilloma. The continuous myoepithelial cell layer shows positive nuclear stating for P63 and cytoplasmic CK5/6 staining (thin arrow). In addition, hyperplastic epithelial cells show a mosaic pattern of cytoplasmic CK5/6 positivity (thick arrow). (b) Atypical papilloma. (bi) H\&E. (bii) P63 and CK5/6. The area with atypical histological features (thin arrow) shows loss of epithelial CK5/6 staining compared with the nonatypical area (thick arrow). (c) Papillary carcinoma. (ci) H\&E. (cii) P63 and CK5/6. Complete absence of P63 and CK5/6 staining indicates absence of the myoepithelial cell layer. Epithelial CK5/6 staining is also absent. (d) Core biopsy of papillary lesion. (di) Broad sclerotic fibrovascular cores on H\&E (arrow). (dii) Positive CK5/6 staining of epithelial cells (arrow). Scale bars $=100 \mu \mathrm{m}$.

\section{Predictive Assessment of Benign Papilloma on Core Biopsy}

To test this, a non-overlapping group of 42 excised papillary lesions that had been diagnosed on initial core biopsy was examined. Fibrovascular cores and epithelial CK5/6 staining were evaluated in core biopsy sections with the presence of broad, sclerotic cores, and any CK5/6 staining used to designate a benign category. This categorization was then compared with the final excision diagnosis for each case (Table 2).

Overall, 27/42 cases (64\%) were allocated to the benign category according to this scheme. This included 25 cases that were diagnosed as benign on excision and the two cases that were ultimately diagnosed as atypical. The 15 cases that were not classified as benign included 13/13 cases with a malignant final diagnosis and 2 benign cases.

The overall misclassification rate was $4 / 42(10 \%)$. Two of 27 benign lesions (7\%) were misclassified and $2 / 2$ atypical cases were assigned to the benign category. All malignant lesions $(n=13)$ were accurately classified. Overall sensitivity of the classifier was $87 \%$ and specificity was $93 \%$. The positive predictive value of this classifier was $87 \%(13 / 15)$ and negative predictive value was 93\% (25/27).

\section{Discussion}

Diagnostic evaluation of papillary lesions of the breast on core biopsy is a challenging area of diagnostic pathology that is especially relevant to 


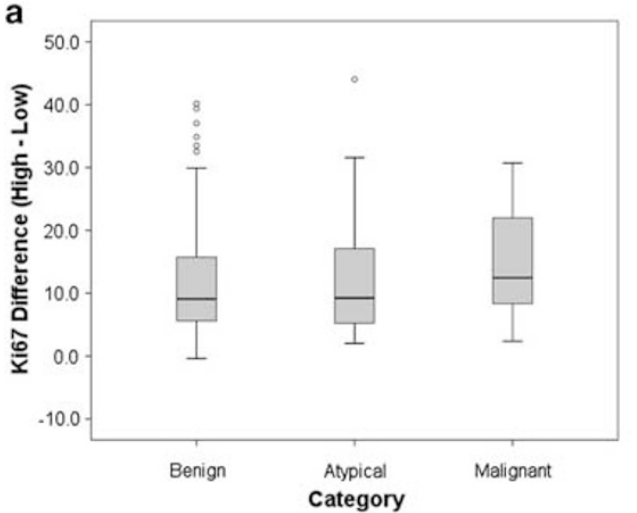

b

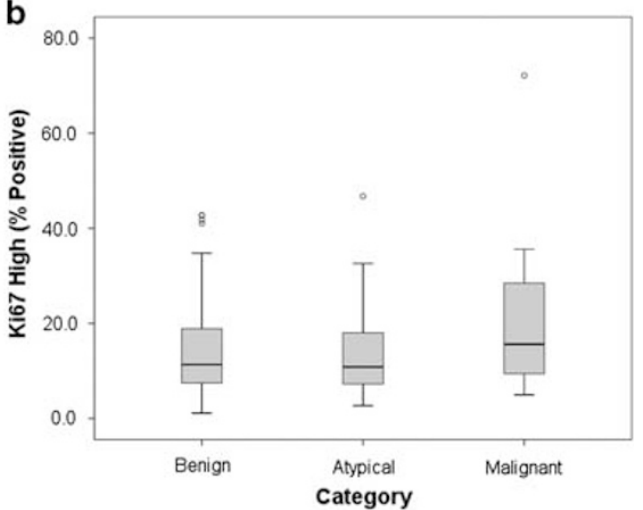

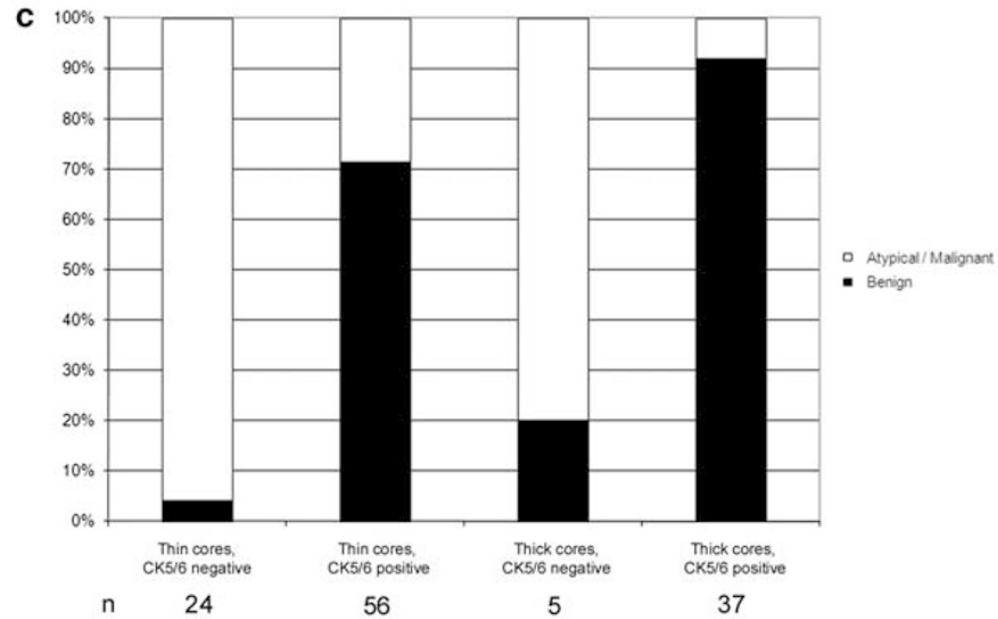

Figure 2 (a) The difference between high and low Ki67 \% positive scores for benign, atypical, and malignant papillary lesions $(n=120$, $P=0.240$, Kruskal-Wallis). (b) High score Ki67 \% positive for benign, atypical, and malignant papillary lesions $(n=120, P=0.173$, Kruskal-Wallis). (c) The proportion of papillary lesions showing combined fibrovascular core and epithelial CK5/6 staining characteristics that are benign versus atypical/malignant $(n=122, P<0.001$, Fisher's exact test).

Table 2 Predicted versus final diagnostic categories for 42 papillary lesions

\begin{tabular}{lrc}
\hline & \multicolumn{2}{c}{ Predicted } \\
\cline { 2 - 3 } & Benign & Not benign \\
\hline Final diagnosis & & \\
$\quad$ Benign & 25 & 2 \\
Atypical & 2 & 13 \\
Malignant & & 13 \\
\hline
\end{tabular}

the assessment of mammographic screen detected lesions. To address the issue of whether benign papillary lesions could be accurately diagnosed on core biopsy, we initially conducted detailed histopathologic review of an inclusive group of 127 excised cases in addition to dual immunohistochemical staining for the basal/myoepithelial cell markers p63 and CK5/6 and the proliferation marker Ki67. The aim was to identify distinctive features of benign papillomas that could form the basis of a predictive classifier applicable to core biopsies.
A feature of papillary lesion pathology, emphasized by this review was striking intralesional heterogeneity. Atypical papillary lesions in particular were characterized by focal atypical architecture as well as focal myoepithelial cell layer defects. In addition, there was focal loss of staining for CK5/6 usually in the atypical areas within the lesion. Cellular proliferation showed marked regional variation in benign, atypical, and malignant papillary lesions, with low and high scores different by up to $44 \%$. These data suggest that asymmetrical growth may be a feature of many papillary lesions. They also show the potential difficulty of accurate assessment of the limited material seen in core biopsy samples.

The detailed catalogue of pathologic characteristics derived from the histological review of excised papillary lesions showed that while there were a number of individual features that were significantly different between benign, atypical, and malignant cases, considerable overlap for many of these features was present across the diagnostic categories. For example, there was a relative shift toward more atypical nuclear features in malignant 
compared with benign lesions; however, intermediate grade nuclei were most common in all categories. Overall, there were no individual features of benign lesions that were sufficiently distinctive for use as a diagnostic indicator. However, over $90 \%$ of lesions showing the combination of broad, sclerotic fibrovascular cores, and epithelial staining for CK5/6 were benign. In view of this result, and also the potential accessibility of these features to evaluation on core biopsy, a putative predictor of a benign papilloma based on these two features was formulated.

In a non-overlapping group of 42 papillary lesions, 27 were assigned to the benign category using the combined assessment of fibrovascular cores and CK5/6 staining. This included two cases that were ultimately diagnosed as atypical in the surgically excised specimen. All 13 cases diagnosed as malignant on excision were accurately classified. Overall, these data suggest that combined assessment of the presence of broad, sclerotic fibrovascular cores, and CK5/6 staining in epithelial cells of papillary lesions can reliably distinguish benign and malignant papillary lesions, but cannot separate benign and atypical cases. The failure to accurately classify atypical lesions may be attributable to a degree of 'sampling error' in core biopsies given the focal nature of the atypia.

The management of women with a core biopsy diagnosis of a benign papilloma remains problematic because, although the majority of these lesions are benign, the reported rate of upgrading to an atypical or malignant final diagnosis is disconcertingly high, leading to a general pragmatic view that all papillary lesions should be excised to establish a definitive diagnosis. ${ }^{4,6,7,9-11}$ This approach, however, results in overtreatment of many women as the small increase in cancer risk associated with a single-benign papilloma is analogous to that of usual ductal hyperplasia. ${ }^{12}$

There have been a number of attempts to develop ancillary methods that may improve the diagnostic accuracy of papillary lesion assessment on core biopsy. For example, several studies have shown that a combination of myoepithelial and CK markers is useful in the classification of papillary lesions. ${ }^{8,13-15}$ In core biopsy specimens, Shah et $a l^{16}$ reported that a panel of immunohistochemical stains (CK5/6, Calponin and P63) improved the mean positive predictive value of a benign diagnosis from 79.2 to $82.1 \%$ in 129 cases examined by four pathologists, compared with $\mathrm{H} \& \mathrm{E}$ sections alone. More recently, Grin et $a{ }^{17}$ reported that immunohistochemical staining for estrogen receptor (ER) and CK5 was useful, with an ER-low/ CK5-high profile on core biopsy supportive of a benign diagnosis.

Clearly, an important caveat for the use of ancillary methods in the diagnosis of papillary lesions is that they must be interpreted in the context of routine histopathologic assessment. This was shown by Grin et $a l^{17}$ who pointed out that the ER-low/CK5 profile they presented as an indicator of a benign papillary lesion may also be seen in the 'basal-like' DCIS, although the morphology would serve to be sufficiently distinctive in this case. Similarly, the classification approach used in this study based on fibrovascular core thickness and CK5/6 staining needs to be interpreted in the context of other histopathological, clinical, and radiological information. However, in a situation where these features favor a benign lesion, the presence of broad, sclerotic fibrovascular cores, and staining for CK5/6 in epithelial cells could provide useful additional input to the ultimately clinical decision of whether to further excise the lesion.

In this study, detailed examination of a group of excised papillary lesions of the breast revealed the difficulty of accurately diagnosing these lesions on core biopsy. In particular, it was clear that the intrinsic heterogeneity of papillary lesions poses a challenge to limited diagnostic sampling. However, the combination of broad, sclerotic fibrovascular cores, and epithelial staining for CK5/6 was identified as a characteristic that could clearly distinguish benign and malignant papillary lesions, although benign and atypical cases were not separated using this approach. Notwithstanding this limitation, combined assessment of fibrovascular cores and CK5/6 staining features could form a useful addition to clinicopathologic assessment.

\section{Acknowledgements}

PJP and RLB acknowledge funding support from the Cancer Institute NSW. RLB is a Cancer Institute NSW Fellow.

\section{Disclosure/conflict of interest}

The authors declare no conflict of interest.

\section{References}

1 Mulligan AM, O’Malley FP. Papillary lesions of the breast: a review. Adv Anat Pathol 2007;14:108-119.

2 Collins LC, Schnitt SJ. Papillary lesions of the breast: selected diagnostic and management issues. Histopathology 2008;52:20-29.

3 Liberman L, Bracero N, Vuolo MA, et al. Percutaneous large-core biopsy of papillary breast lesions. AJR Am J Roentgenol 1999;172:331-337.

4 Mercado CL, Hamele-Bena D, Oken SM, et al. Papillary lesions of the breast at percutaneous core-needle biopsy. Radiology 2006;238:801-808.

5 Sydnor MK, Wilson JD, Hijaz TA, et al. Underestimation of the presence of breast carcinoma in papillary lesions initially diagnosed at core-needle biopsy. Radiology 2007;242:58-62.

6 Tseng HS, Chen YL, Chen ST, et al. The management of papillary lesion of the breast by core needle biopsy. Eur J Surg Oncol 2009;35:21-24. 
7 Rizzo M, Lund MJ, Oprea G, et al. Surgical follow-up and clinical presentation of 142 breast papillary lesions diagnosed by ultrasound-guided core-needle biopsy. Ann Surg Oncol 2008;15:1040-1047.

8 Tse GM, Tan PH, Moriya T. The role of immunohistochemistry in the differential diagnosis of papillary lesions of the breast. J Clin Pathol 2009;62: 407-413.

9 Skandarajah AR, Field L, Yuen Larn Mou A, et al. Benign papilloma on core biopsy requires surgical excision. Ann Surg Oncol 2008;15:2272-2277.

10 Bode MK, Rissanen T, Apaja-Sarkkinen M. Ultrasonography-guided core needle biopsy in differential diagnosis of papillary breast tumors. Acta Radiol 2009;50:722-729.

11 Bernik SF, Troob S, Ying BL, et al. Papillary lesions of the breast diagnosed by core needle biopsy: 71 cases with surgical follow-up. Am J Surg 2009;197:473-478.

12 Lewis JT, Hartmann LC, Vierkant RA, et al. An analysis of breast cancer risk in women with single, multiple, and atypical papilloma. Am J Surg Pathol 2006;30: 665-672.
13 de Moraes Schenka NG, Schenka AA, de Souza Queiroz L, et al. Use of p63 and CD10 in the differential diagnosis of papillary neoplasms of the breast. Breast J 2008;14:68-75.

14 Douglas-Jones A, Shah V, Morgan J, et al. Observer variability in the histopathological reporting of core biopsies of papillary breast lesions is reduced by the use of immunohistochemistry for CK5/6, calponin and p63. Histopathology 2005;47:202-208.

15 Ichihara S, Fujimoto T, Hashimoto K, et al. Double immunostaining with p63 and high-molecular-weight cytokeratins distinguishes borderline papillary lesions of the breast. Pathol Int 2007;57:126-132.

16 Shah VI, Flowers CI, Douglas-Jones AG, et al. Immunohistochemistry increases the accuracy of diagnosis of benign papillary lesions in breast core needle biopsy specimens. Histopathology 2006;48:683-691.

17 Grin A, O’Malley FP, Mulligan AM. Cytokeratin 5 and estrogen receptor immunohistochemistry as a useful adjunct in identifying atypical papillary lesions on breast needle core biopsy. Am J Surg Pathol 2009;33: $1615-1623$. 